\title{
Decreased plasma musclin levels are associated with potential atrial fibrillation in non-diabetic patients
}

\author{
Yuan Zhong", Jingying Zhang", Kai Tang, Wenxin Kou, Shaojie Xu, Haotian Yang, Lu Liu, Peipei Luan, \\ Abdul-Quddus Mohammed, Fuad A. Abdu, Dongdong Zhao, Hailing Li^, Wenhui Peng, Yawei Xu \\ Department of Cardiology, Shanghai Tenth People's Hospital, Tongji University School of Medicine, Shanghai, China \\ Contributions: (I) Conception and design: Y Zhong, J Zhang; (II) Administrative support: D Zhao, H Li; (III) Provision of study materials or patients: \\ K Tang, S Xu, W Peng, Y Xu, ; (IV) Collection and assembly of data: W Kou, P Luan, H Yang, L Liu; (V) Data analysis and interpretation: Y Zhong, \\ J Zhang, H Li; (VI) Manuscript writing: All authors; (VII) Final approval of manuscript: All authors. \\ \#These authors contributed equally to this work. \\ Correspondence to: Hailing Li, MD, PhD; Dongdong Zhao, MD, PhD. Department of Cardiology, Shanghai Tenth People's Hospital, Tongji \\ University School of Medicine, 301 Yanchang Road, Shanghai 200072, China. Email: 1986lihailing@tongji.edu.cn; 249620601@163.com.
}

Background: Musclin is involved in the regulation of natriuretic peptide (NP) clearance and may affect the concentration of atrial natriuretic peptide (ANP). It has also been found to play an important role in several diseases, such as diabetes mellitus and hypertension. Both abnormalities in ANP and associated medical history are involved in the pathogenesis of atrial fibrillation (AF). However, plasma concentration of musclin as a biomarker for risk stratification in patients with AF has not been fully investigated.

Methods: Plasma musclin levels were measured in 290 patients with AF (including 199 paroxysmal AF patients and 91 persistent AF patients) and 120 control subjects. The association between plasma musclin levels and AF onset, as well as its predictive effects on clinical outcomes after cryoballoon ablation were analyzed.

Results: AF patients were found to have a lower concentration of plasma musclin than healthy controls. Moreover, in the non-diabetic group and normal N-terminal pro-brain natriuretic peptide (NT-proBNP) level group, the association between lower plasma concentration of musclin and AF remained significant. According to receiver operating characteristic (ROC) curve analysis, the optimal cut-off point of musclin for predicting $\mathrm{AF}$ onset was $54.94 \mathrm{ng} / \mathrm{mL}$, which had a sensitivity of $81.67 \%$ and a specificity of $31.47 \%$ [area under the ROC curve $(\mathrm{AUC})=60.71 \%$ ]. In follow-up studies, both diabetes and left atrial size were independent predictors of $\mathrm{AF}$ recurrence after ablation, while musclin showed only a modest relationship with the outcome of cryoballoon ablation.

Conclusions: Our data indicated that decreased musclin was associated with the onset of AF. Moreover, lower plasma levels of musclin were an independent risk factor of AF in non-diabetic patients. Our studies suggest that musclin could be a predictive factor for the onset of $\mathrm{AF}$.

Keywords: Atrial fibrillation (AF); biomarkers; musclin; natriuretic peptides (NP)

Submitted Apr 13, 2020. Accepted for publication Nov 01, 2020.

doi: $10.21037 / \mathrm{atm}-20-3259$

View this article at: http://dx.doi.org/10.21037/atm-20-3259

\footnotetext{
$\wedge$ ORCID: 0000-0002-9903-3683.
} 


\section{Introduction}

Atrial fibrillation $(\mathrm{AF})$ is one of the most common types of arrhythmias. It affects $1-2 \%$ of the population worldwide and is a major cause of morbidity, mortality, and healthcare expenditure (1). However, the detection and diagnosis of suspected AF patients is challenging.

A considerable proportion of patients first present with severe complications, such as stroke and heart failure (2), but not with $\mathrm{AF}$ symptoms or a definitive $\mathrm{AF}$ medical history (3). Moreover, AF is often paroxysmal and cannot be easily detected by a single electrocardiogram (ECG). Despite continuous patch monitoring, some patients with mild symptoms are still easily missed (4). Further, determining the optimal AF treatment can be especially difficult. Although rhythm control and anti-coagulation treatments are based mostly on oral medications, catheter ablation and other surgical treatments can also improve prognosis (1). To date, early reporting of biomarkers has aided in the clinical detection of high-risk AF. A medical history of high blood pressure, obesity, or diabetes (5), and elevation of natriuretic peptides (NPs), such as C-reactive protein and interleukin-6 (6), have been identified as components of risk stratification.

Musclin, also referred to as osteocrin, was originally identified as a secretory peptide from bone and muscles $(7,8)$, and its expression has been detected in cardiac muscles (9). It has also been found to be related to both cardiovascular and metabolic diseases. Musclin has a well-conserved homology with NP members but no natriuretic activity. It binds to natriuretic peptide receptor 3 (NPR3), which is a clearance receptor for atrial, B-type, and C-type natriuretic peptides (ANP, BNP, and CNP) (10). Studies have reported that musclin binds to NPR3 competitively with ANP and increases ANP concentrations in a local or systemic manner (11). Another study reported that musclin exerts its physiological function by enhancing ANP-dependent signaling (10). This study also found that overexpression of musclin can suppress the worsening of chronic heart failure (CHF) after myocardial infarction (MI) by inhibiting clearance of NP family members. A series of studies have reported the relationship of ANP or BNP with AF (12-15), and NP has been recommended as a tool for the routine evaluation of patients with $\mathrm{AF}$ (16). Accordingly, physical exercise can up-regulate circulating musclin (17) and reduce the risk of atrial arrhythmias (18). However, musclin is found to increase insulin resistance in obesity (19) and vasoconstriction (20), which has been suggested to play a role in the onset of AF. However, to date, plasma musclin concentration as a potential biomarker in $\mathrm{AF}$ patients or as a measure of the efficacy of catheter ablation has not been investigated.

Therefore, the current study aimed to identify the association between circulating musclin and the trend of $\mathrm{AF}$ onset, as well as clinical outcomes after cryoballoon ablation. The following article is presented in accordance with the Strengthening the Reporting of Observational Studies in Epidemiology (STROBE) reporting checklist (available at http://dx.doi.org/10.21037/atm-20-3259).

\section{Methods}

\section{Study population}

This observational study enrolled 290 non-valvular AF patients and 120 non-AF controls from the Department of Cardiology, Shanghai Tenth People's Hospital, China. Patients with AF were diagnosed according to criteria described in the AF guidelines (21). All participants were admitted between March 2016 and August 2017. The exclusion criteria were: (I) acute or chronic infections or inflammatory diseases, severe liver or renal dysfunction, malignant tumors, hematologic disorders, or a history of cerebral infarction or transient cerebral ischemia within 6 months before this study; (II) reversible AF due to acute alcoholism, cardiopulmonary surgery, endocrine abnormalities and so on; (III) myocarditis or cardiomyopathies; (IV) significant valvular disease, which was defined as greater than grade II; (V) recent interventional treatment of acute coronary events; (VI) previous left atrial ablation or cardiac surgery; and (VII) New York Heart Association (NYHA) class IV heart failure or left ventricular ejection fraction (LVEF) $<40 \%$.

Our study complied with the Declaration of Helsinki (as revised in 2013) and was approved by the ethical review board of the hospital (Shanghai Tenth People's Hospital Affiliated to Tongji University School of Medicine, Shanghai, China; No. 20K60). Written informed consent was obtained from all subjects before their participation in the study. A copy of the written consent is available for review from the Editor-in-Chief of this journal.

\section{Clinical characteristics}

Paroxysmal AF was defined as AF terminating spontaneously or with intervention within 7 days of onset. Persistent AF was defined as continuous AF lasting 7 days or more, or beyond 
7 days, in which episodes were terminated by cardioversion, either with drugs or with direct current cardioversion. Coronary artery disease (CAD) was defined as the occurrence of exercise- or stress-related chest symptoms due to narrowing of $\geq 70 \%$ in one or more of the major coronary arteries or $\geq 50 \%$ in the left main coronary artery (22).

Left atrium diameter (LAD) and left ventricular ejection fraction (LVEF) were measured through transthoracic ultrasonic testing. Heart failure was considered when a patient had NYHA class IV heart failure (23) or a LVEF of $<40 \%$ (24). Body mass index (BMI) was calculated as weight divided by the square of height. Hypertension was defined as blood pressure $>140 / 90 \mathrm{mmHg}$ or the use of antihypertensive medications. Diabetes was diagnosed according to the World Health Organization (WHO) criteria. Risk scores for stroke were calculated using the $\mathrm{CHA}_{2} \mathrm{DS}_{2}$-VASc Score (8). Cryoballoon ablation was performed by qualified physicians in $123 \mathrm{AF}$ patients with a standard ablation procedure which has been previously reported in detail (25).

\section{Laboratory tests, electrocardiogram, and transthoracic echocardiogram}

A comprehensive clinical assessment was carried out. Vascular disease was identified through a previously established diagnosis of CAD, peripheral artery disease, carotid disease, renal artery disease, or aortic atheroma. Standard 12-lead electrocardiogram and echocardiography were performed on all subjects. All transthoracic echocardiogram (TTE) parameters were obtained using Vivid E9, GE Vingmed Ultrasound AS, Norway. In all groups, venous blood samples were collected in tubes with ethylenediaminetetraacetic acid (EDTA) and centrifuged at $1,000 \mathrm{~g}$ for $10 \mathrm{~min}$. Plasma was stored in a $-80{ }^{\circ} \mathrm{C}$ ultra-low temperature refrigerator until biochemical analysis. The levels of plasma musclin were quantified using enzymelinked immunoabsorbent assays (ELISA). Briefly, the wells of a polyvinyl chloride (PVC) microtiter plate was coated with $50 \mu \mathrm{g} / \mathrm{mL}$ of the capture antibody (sc-365631, Santa Cruz) and incubated overnight at $4{ }^{\circ} \mathrm{C}$. The remaining protein-binding sites were blocked using $200 \mu \mathrm{L}$ blocking buffer (5\% non-fat dry milk/PBS) per well. Diluted samples $(100 \mu \mathrm{L})$ were added to each well. Standards (ab73487, Abcam) and blanks were run with each plate and incubated for $90 \mathrm{~min}$ at $37^{\circ} \mathrm{C}$. A horseradish peroxidase (HRP)conjugated detector antibody (sc-99096, Santa Cruz) against the target was then applied. All samples were assayed in duplicate, and values were normalized to a standard curve.

\section{Follow-up study}

One hundred and twenty-three AF patients underwent cryoballoon ablation and were monitored during their hospital stay after the procedure (the first 24-48 hours) with ECG and Holter studies. After hospital discharge, patients who accepted cryoballoon ablation were scheduled for visits in the outpatient clinics at 1, 3, 6, and 12 months after cryoballoon ablation, or earlier if symptoms consistent with recurrent $\mathrm{AF}$ developed after the ablation. Rapid recurrence of atrial arrhythmias was determined using 24-hour Holter monitoring and 12-lead ECG. After a blanking period of 3 months, recurrence of AF was defined as any recording of AF on ECG or an episode longer than $30 \mathrm{~s}$ on the 24-hour ECG Holter registration. Atrial tachycardia (AT) was defined as a regular, organized supraventricular rhythm at a constant atrial rate of $\geq 100$ beats $/ \mathrm{min}$. All early and late recurrences were classified as AF or AT, depending on the morphology and regularity of the rhythm.

\section{Statistical analysis}

Statistical analyses were performed using Statistical Package for Social Sciences (SPSS) for Windows 10. Numerical variables with a normal distribution were expressed as the mean $\pm \mathrm{SD}$, and numerical variables with a skewed distribution were expressed as the median and interquartile ranges (IQRs). Categorical variables were expressed as percentages. For numerical variables, an independent sample $t$ test and Mann-Whitney $\mathrm{U}$ test were used for intergroup comparisons. Chi-square and Fisher's exact tests were used to compare categorical variables. The correlations between musclin and other clinical parameters were analyzed with Spearman's rank correlation test. To obtain the optimal cut-off points of musclin concentrations for predicting $\mathrm{AF}$ onset, receiver-operating characteristic (ROC) curve analysis was performed, and the area under the curve (AUC) was calculated. Those independent predictors derived from logistic regression were selected and incorporated in logistic regression models. A twotailed $\mathrm{P}<0.05$ was considered statistically significant for all analyses. For multiple comparisons, Bonferroni adjustment was used to correct $\mathrm{P}$ values to $<0.025$.

\section{Results}

\section{Subject characteristics}

The study population had a mean age of $64.14 \pm 9.36$ years, 
Table 1 Baseline characteristics of the study population

\begin{tabular}{|c|c|c|c|c|c|c|}
\hline Clinical characteristics (total $n=410$ ) & Control $(n=120)$ & $A F(n=290)$ & $P$ value & Paroxysmal AF $(n=199)$ & Persistent AF $(n=91)$ & $P$ value \\
\hline \multicolumn{7}{|l|}{ Baseline characteristics } \\
\hline Male, n (\%) & $53(44.17)$ & $165(56.90)$ & 0.019 & $108(54.27)$ & $57(62.64)$ & 0.182 \\
\hline Age, years & $62.3 \pm 7.5$ & $64.63 \pm 9.04$ & 0.096 & $64.5 \pm 9.6$ & $66.6 \pm 7.7$ & 0.079 \\
\hline BMI $\left(\mathrm{kg} / \mathrm{m}^{2}\right)$ & $23.3 \pm 2.7$ & $25.07 \pm 3.59$ & 0.001 & $25.14 \pm 3.43$ & $24.93 \pm 3.93$ & 0.687 \\
\hline Hypertension & $33(27.50)$ & $179(61.72)$ & $<0.001$ & $109(54.77)$ & 70 (76.92) & $<0.001$ \\
\hline Diabetes mellitus & $11(9.17)$ & $44(15.17)$ & 0.104 & $28(14.07)$ & $16(17.58)$ & 0.439 \\
\hline \multicolumn{7}{|l|}{ Medications before ablation, $\mathrm{n}(\%)$} \\
\hline Amiodarone therapy & $0(0.0)$ & $61(21.03)$ & $<0.001$ & $56(28.14)$ & $5(5.50)$ & $<0.001$ \\
\hline \multicolumn{7}{|l|}{ Echocardiographic parameters } \\
\hline LAD (mm) & $38.05 \pm 5.55$ & $41.41 \pm 5.29$ & $<0.001$ & $39.99 \pm 5.13$ & $44.52 \pm 4.23$ & $<0.001$ \\
\hline LVEF (\%) & $66.81 \pm 5.70$ & $63.17 \pm 5.72$ & $<0.001$ & $64.31 \pm 5.18$ & $60.78 \pm 6.08$ & $<0.001$ \\
\hline \multicolumn{7}{|l|}{ Baseline plasma level } \\
\hline NT-proBNP (pg/mL) & $\begin{array}{c}64.25 \\
{[36.90,120.08]}\end{array}$ & $\begin{array}{c}302.90 \\
{[122.60,796.60]}\end{array}$ & 0.001 & $\begin{array}{c}183.70 \\
{[83.35,534.0]}\end{array}$ & $\begin{array}{c}800.2 \\
{[422.88,1,053.75]}\end{array}$ & $<0.001$ \\
\hline TGF- $\beta$ (ng/mL) & $11.05 \pm 2.33$ & $9.93 \pm 3.88$ & 0.003 & $9.99 \pm 3.87$ & $9.81 \pm 3.91$ & 0.725 \\
\hline Musclin (ng/mL) & $\begin{array}{c}82.83 \\
{[60.52,155.65]}\end{array}$ & $\begin{array}{c}70.40 \\
{[50.57,101.32]}\end{array}$ & $<0.001$ & $\begin{array}{c}69.30 \\
{[50.98,103.91]}\end{array}$ & $\begin{array}{c}73.13 \\
{[49.76,98.33]}\end{array}$ & 0.684 \\
\hline
\end{tabular}

Numerical variables with a normal distribution were presented as the mean \pm SD and numerical variables with a skewed distribution were presented as the median [interquartile range] and categorical variables presented as percentages. AF, atrial fibrillation; BMI, body mass index; LAD, left atrial diameter; LVEF, left ventricular ejection fraction; NT-proBNP, N-terminal pro-brain natriuretic peptide.

and 218 participants $(53.17 \%)$ were male. Other demographic and clinical data are presented in Table 1. Patients with AF had higher BMI $(25.07 \pm 3.59$ vs. $23.3 \pm 2.7$, $\mathrm{P}=0.001)$ and a higher prevalence of hypertension $(61.72 \%$ vs. $27.50 \%, \mathrm{P}<0.001)$ compared to the controls. Significant differences were also observed in LAD and LVEF between the AF and non-AF groups, which were $41.41 \pm 5.29$ vs. $38.05 \pm 5.55 \mathrm{~mm}(\mathrm{P}<0.001)$ and $63.17 \% \pm 5.72 \%$ vs. $66.81 \% \pm 5.70 \%(\mathrm{P}<0.001)$, respectively. The level of $\mathrm{N}$-terminal pro-brain natriuretic peptide (NT-proBNP) was higher in AF patients compared to non-AF patients, while transforming growth factor (TGF)- $\beta$ showed the opposite trend $(\mathrm{P}=0.001$ and $\mathrm{P}=0.003$, respectively). Compared to paroxysmal AF patients, univariate analysis showed that the persistent AF group had a higher prevalence of hypertension, received less drug (amiodarone and propafenone) therapy, and had a larger LAD diameter, reduced LVEF, and higher plasma levels of NT-proBNP (Table 1).

\section{Decreased plasma musclin levels in AF patients independently increases $A F$ risk}

The mean plasma musclin levels in the entire study cohort were $100.03 \pm 83.78 \mathrm{ng} / \mathrm{mL}$, with an interquartile range from $53.72-107.30 \mathrm{ng} / \mathrm{mL}$. Musclin levels differed significantly depending on the clinical group, with a gradient from the control group $(126.08 \pm 103.48 \mathrm{ng} / \mathrm{mL})$ to the AF cohort $(89.11 \pm 71.40 \mathrm{ng} / \mathrm{mL})(\mathrm{P}<0.001$, Figure $1 A)$. When the subjects were divided according to medical history of diabetes mellitus and hypertension, plasma musclin levels of $\mathrm{AF}$ patients were found to be decreased in non-diabetes 
A

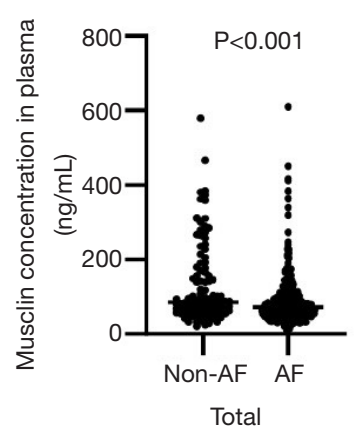

C

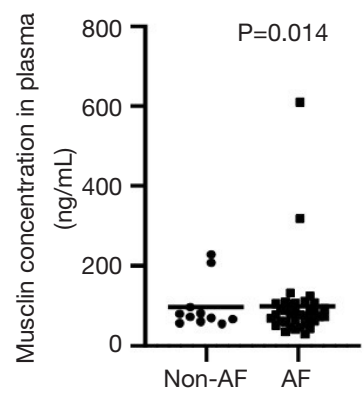

Hypertension group

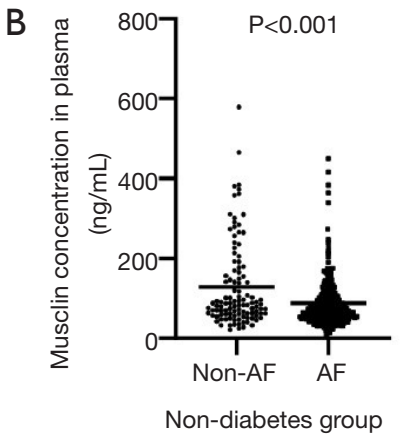

D

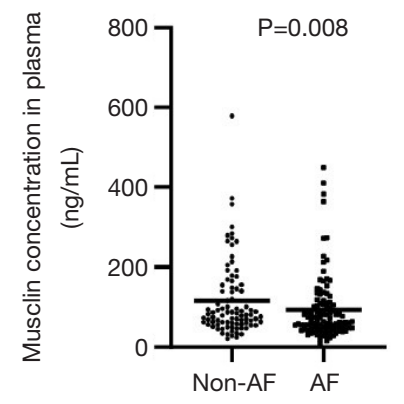

Non-hypertension group

\section{$E$}

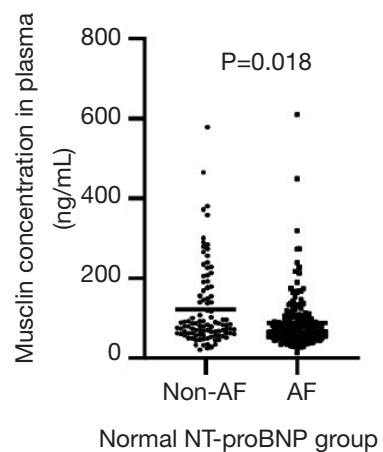

Figure 1 Musclin concentrations in different groups. (A) The plasma musclin concentration in control group (126.08 $\pm 103.48 \mathrm{ng} / \mathrm{mL})$ was significantly higher than it in AF cohort $(89.11 \pm 71.40 \mathrm{ng} / \mathrm{mL})$ in the entire study group. (B) In non-diabetic patients, the relationship between musclin and AF remained significant. When patients were divided into the hypertension (C) and non-hypertension (D) groups, musclin was still associated with AF. (E) In patients with normal NT-proBNP (below $500 \mathrm{pg} / \mathrm{mL}$ ), musclin levels were still associated with $\mathrm{AF}$ onset.

group, hypertension and non-hypertension group $(\mathrm{P}<0.001$, $\mathrm{P}=0.008$ and $\mathrm{P}=0.014$, respectively; Figure $1 B, C, D)$. In the normal NT-proBNP group, the association between musclin and AF remained strongly significant $(\mathrm{P}=0.018$, Figure 1E).

Plasma musclin levels were found to be negatively correlated with $\mathrm{AF}$ in the entire cohort, the non-diabetic group, and the normal NT-proBNP group (Table 2). In multivariate analyses, after adjustment for potential confounders in the non-diabetic group (sex, age, BMI, $\mathrm{LAD}, \mathrm{LVEF}$, and TGF- $\beta$ ), the relationship between musclin and $\mathrm{AF}$ onset remained significant $(\mathrm{P}=0.011$, Table 3). 
Table 2 Correlations between musclin and other variables

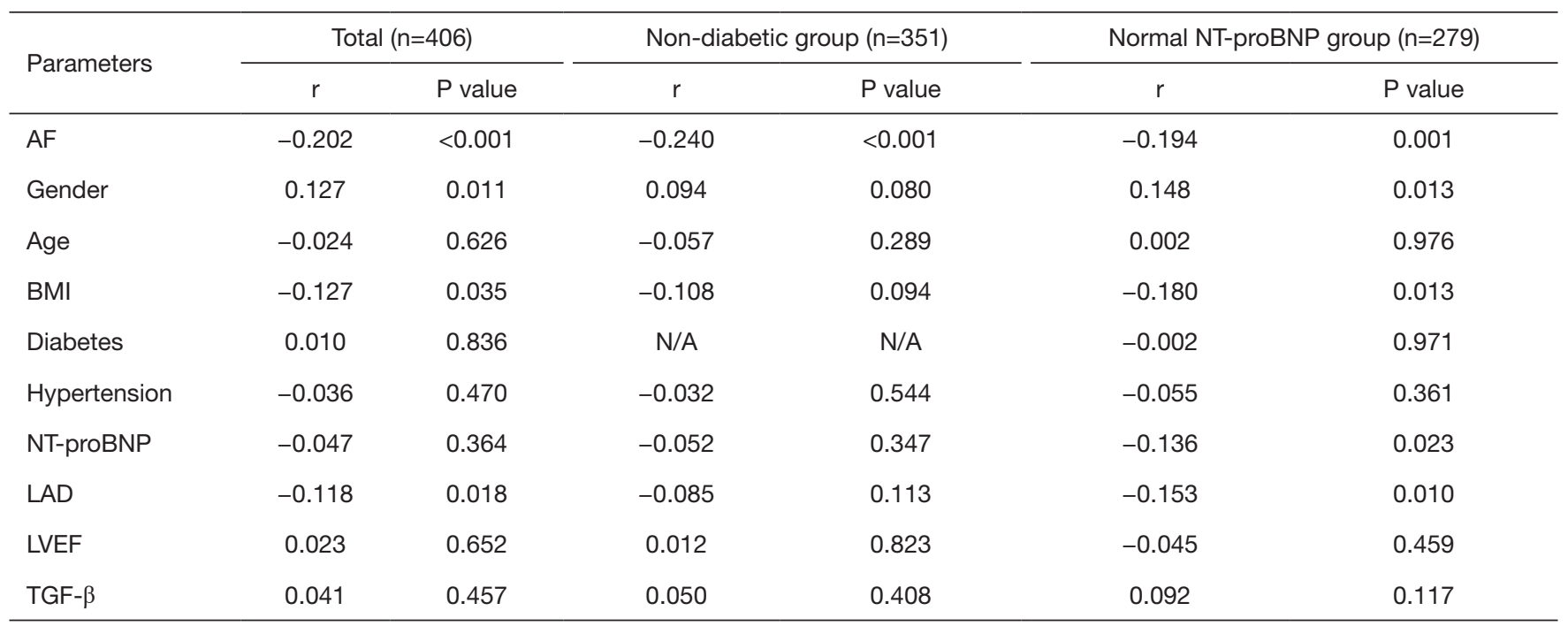

AF, atrial fibrillation; BMI, body mass index; LAD, left atrial diameter; LVEF, left ventricular ejection fraction; NT-proBNP, N-terminal probrain natriuretic peptide.

Table 3 Univariate and multiple variation analysis of musclin and AF in non-diabetic groups

\begin{tabular}{lcccccc}
\hline Covariations & P value & OR & $95 \% \mathrm{Cl}$ & P adj & OR-adj & 95\% Cl adj \\
\hline Gender & 0.099 & 0.683 & $0.434-1.074$ & 0.481 & 0.775 & $0.381-1.574$ \\
Age & 0.080 & 1.021 & $0.998-1.046$ & 0.662 & 0.990 & $0.948-1.035$ \\
BMI & 0.009 & 1.130 & $1.031-1.239$ & 0.743 & 1.021 & $0.902-1.156$ \\
LAD & $<0.001$ & 1.109 & $1.061-1.160$ & $<0.001$ & 1.169 & 0.929 \\
LVEF & $<0.001$ & 0.903 & $0.862-0.945$ & 0.075 & $0.858-1.007$ \\
TGF- $\beta$ & 0.012 & 0.904 & $0.835-0.978$ & 0.164 & 0.928 & $0.835-1.031$ \\
Musclin & $<0.001$ & 0.994 & $0.991-0.997$ & 0.011 & 0.994 & $0.989-0.998$ \\
\hline
\end{tabular}

BMI, body mass index; LAD, left atrial diameter; LVEF, left ventricular ejection fraction.

According to ROC curve analysis, a cut-off point of $54.94 \mathrm{ng} / \mathrm{mL}$ of musclin had a sensitivity of $81.67 \%$ and a specificity of $31.47 \%$ (AUC $=60.71 \%$ ) for predicting the onset of AF (Figure 2A). In non-diabetic patients, the optimal cut-off point of musclin was $55.64 \mathrm{ng} / \mathrm{mL}$, which had a sensitivity of $79.81 \%$ and specificity of $33.47 \%$ (AUC $=61.99 \%$, Figure $2 B$ ).

\section{Follow up: musclin plasma levels have no apparent relationship with clinical outcome after cryoballoon ablation in AF patients}

A total of $123 \mathrm{AF}$ patients underwent cryoballoon ablation. During a mean follow-up period of $15.1 \pm 2.8$ months, 80 patients remained in sinus rhythm without antiarrhythmic drugs (AADs), while 32 patients had AF recurrence (the loss to follow-up rate was $8.94 \%$ ). According to our analysis, a medical history of diabetes $(\mathrm{P}=0.023)$ and $\mathrm{LAD}(\mathrm{P}=0.016)$ were statistically associated with AF recurrence, while musclin showed no significant difference between the recurrence and non-recurrence group $(\mathrm{P}=0.487)$.

\section{Discussion}

This case-control study demonstrated that AF patients without structural heart disease tend to have lower plasma musclin concentrations compared to healthy controls in sinus rhythm. According to ROC curve, levels of plasma 

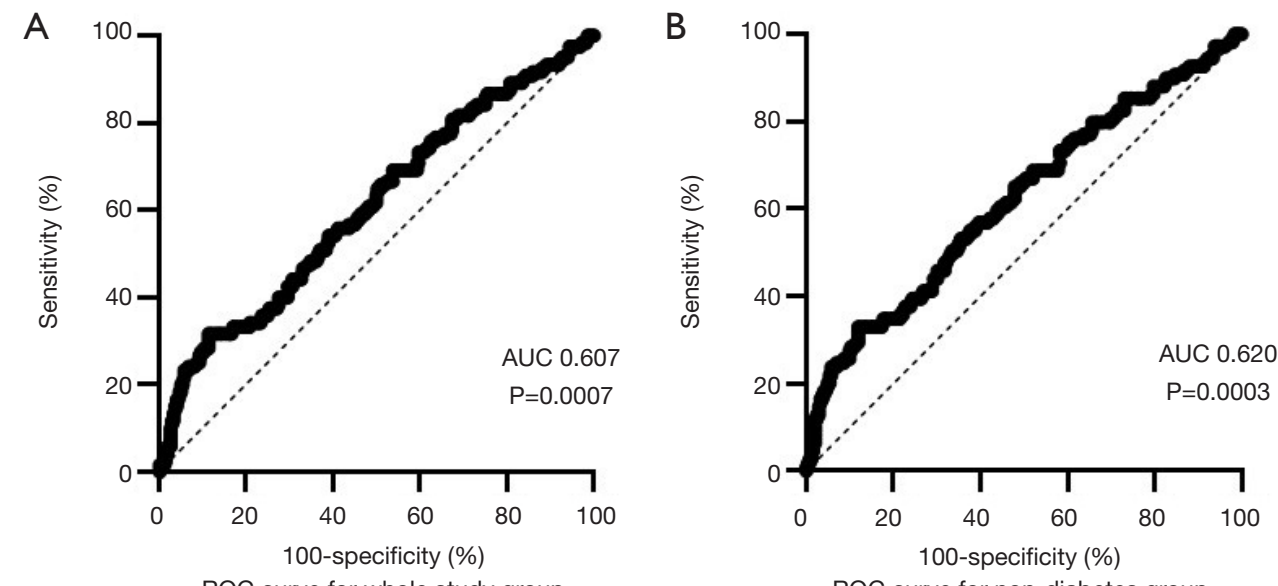

Figure 2 (A) In the entire study group, a cut-off point of $54.94 \mathrm{ng} / \mathrm{mL}$ of musclin had a sensitivity of $81.67 \%$ and a specificity of $31.47 \%$. (B) In the non-diabetic group, the optimal cut-off point for musclin was $55.64 \mathrm{ng} / \mathrm{mL}$, which had a sensitivity of $79.81 \%$ and a specificity of $33.47 \%$.

musclin below $54.94 \mathrm{ng} / \mathrm{mL}$ were sensitive (at a sensitivity of $81.67 \%$ ) to identifying potential AF. Further, musclin independently increased the risk of $\mathrm{AF}$ in the non-diabetic cohort.

Musclin is an activity-stimulated myokine (17) secreted by skeletal muscles (26) that regulates muscle growth and metabolism. Its expression decreases in fasted and insulindeficient conditions (8) and is upregulated when nutrition changes, such as during treatment by insulin (27), palmitate $(28,29)$, and glucosamine. Also, musclin contains two KKKR putative serine protease cleavage sites and a region homologous to members of the NP family (8). It binds to NPR3 competitively with NPs, a clearance receptor for NPs, ANP, BNP, and CNP $(10,11,30)$. Although musclin has no natriuretic activity, it has been shown to attenuate the clearance of NPR-C and increase the availability of NPs $(10,11)$.

Robust studies have demonstrated a relationship between ANP and the development of AF (12-15). A frameshift mutation in the ANP gene was identified in patients with familial $\mathrm{AF}$, and its frameshift product was shown to exhibit modified proteolytic degradation and a markedly longer half-life than ANP $(14,15,31)$, indicating that abnormalities in ANP are involved in the pathogenesis of AF. Previous studies also found that elevated NT-proBNP level independently predicts an increased risk of development of $\mathrm{AF}(32,33)$ and that $\mathrm{BNP}$ independently predicts new onset of AF in ST-segment elevation myocardial infarction (MI) patients (9). Moreover, musclin was found to suppress the worsening of chronic heart failure (CHF) after MI through inhibiting the clearance of NP family peptides (34). It has also been shown to activate the peroxisome proliferators-activated receptor $\mathrm{y}$ (PPAR $\mathrm{P}$ ) pathway in white adipose tissue (35), and it benefits cardiovascular health by acting as a latent protective factor in heart failure (36) and remote ischemic conditioning (37). Physical activity can enhance musclin expression in both muscles and the circulation, which improves exercise endurance (17). Some studies also reported that proper exercise could improve cardiorespiratory fitness in AF patients and prevent atrial and ventricular arrhythmias $(18,38)$. Given this background, we speculated that decreased musclin levels would likely be involved in the occurrence and progression of AF. Other studies have also reported a relationship between musclin and risk factors for $\mathrm{AF}$, such as diabetes and hypertension. Musclin increases insulin resistance, and increased musclin in the circulation is associated with the onset of diabetes $(19,39)$. Also, circulating musclin has been shown to induce vascular constriction and the onset of hypertension (20). Therefore, predicting the function of musclin is complicated.

Our study found plasma musclin levels to be negatively correlated with the onset of AF. Moreover, to exclude confounding bias, our study cohort was divided into different groups. In the non-diabetic group, the association between musclin and AF remained significant and musclin was revealed to be an independent predictor of AF. In patients with and without hypertension, musclin was associated with AF development. Furthermore, musclin 
also had a negative correlation with $\mathrm{AF}$ in patients with normal plasma levels of NT-proBNP. Hence, musclin could help to evaluate the risk of $\mathrm{AF}$ in patients who do not have traditional risk factors such as hypertension, diabetes and heart failure.

Cryoballoon ablation has become an effective therapy for patients with drug-refractory AF (40). In addition, the identification of pre-ablation predictors of AF recurrence would help to improve ablation outcomes, and avoid unnecessary procedures and related complications. In the present study, musclin levels did not differ between the AF recurrence and non-recurrence groups. However, LAD and a medical history of diabetes were found to be associated with AF relapse after cryoballoon ablation, which is consistent with previous studies $(41,42)$. It is difficult to accurately determine the risk factors involved in the permanent elimination of AF due to its complex mechanisms. The outcomes of AF elimination vary depending on several factors, including, but not limited to, the type of AF, cardiac substrate, duration of follow-up, and the extent of monitoring during follow-up (43). Previous investigations with long-term follow-up found that higher levels of NT-proBNP were correlated with AF recurrence $(44,45)$. It has been speculated that the levels of musclin in the AF recurrence group influence plasma NPs levels. However, due to insufficient follow-up time and the small sample size in our study, the relationship between musclin and outcomes of cryoballoon ablation remain uncertain.

Several limitations of the present study should be considered. The major limitations are the relatively small sample size, insufficient follow-up period, and differences in patient characteristics, all of which may have contributed to the lack of significant difference observed between the recurrence and non-recurrence groups $(\mathrm{P}=0.487)$. Our study was a single-center study; therefore, the results may not be applicable to broader $\mathrm{AF}$ populations, including patients with a history of structural heart disease or diabetes. Secondly, AF recurrence may be underestimated because asymptomatic $\mathrm{AF}$ episodes may require continuous monitoring for 7 to 10 days. In our study, the evaluation of AF recurrence was limited by regular electrocardiogram, 24-hour Holter monitoring, and short-term follow-up (1 year after ablation). Future studies will be required to address the question of whether decreased musclin is a cause or a consequence of AF.

\section{Conclusions}

Our study demonstrated that plasma musclin concentrations were decreased in AF patients without coexisting structural heart disease. Musclin concentrations below $54.94 \mathrm{ng} / \mathrm{mL}$ were found to be sensitive to identifying potential AF. Specifically, we showed that musclin levels provide an independent evaluation for the prediction of AF in nondiabetic patients. Hence, musclin could be considered a predictive factor in the onset of $\mathrm{AF}$.

\section{Acknowledgments}

Funding: This work was supported by Chinese National Natural Science Foundation No. 81670230, 81700291, 81770226, and Fundamental Research Funds for Central Universities No. 1501219105.

\section{Footnote}

Reporting Checklist: The authors have completed the STROBE reporting checklist. Available at http://dx.doi. org/10.21037/atm-20-3259

Data Sharing Statement: Available at http://dx.doi. org/10.21037/atm-20-3259

Conflicts of Interest: All authors have completed the ICMJE uniform disclosure form (available at http://dx.doi. org/10.21037/atm-20-3259). The authors have no conflicts of interest to declare.

Ethical Statement: The authors are accountable for all aspects of the work in ensuring that questions related to the accuracy or integrity of any part of the work are appropriately investigated and resolved. Our study complied with the Declaration of Helsinki (as revised in 2013) and was approved by the ethical review board of the hospital (Shanghai Tenth People's Hospital Affiliated to Tongji University School of Medicine, Shanghai, China; No. 20K60). Written informed consent was obtained from all subjects before their participation in the study. A copy of the written consent is available for review from the Editorin-Chief of this journal.

Open Access Statement: This is an Open Access article distributed in accordance with the Creative Commons Attribution-NonCommercial-NoDerivs 4.0 International License (CC BY-NC-ND 4.0), which permits the noncommercial replication and distribution of the article with the strict proviso that no changes or edits are made and the 
original work is properly cited (including links to both the formal publication through the relevant DOI and the license). See: https://creativecommons.org/licenses/by-nc-nd/4.0/.

\section{References}

1. Zimetbaum P. Atrial Fibrillation. Ann Intern Med 2017;166:ITC33-48.

2. Mozaffarian D, Benjamin EJ, Go AS, et al. Heart Disease and Stroke Statistics-2015 Update: A Report from the American Heart Association. Circulation 2016;133:e38-360.

3. Lavandero S, Troncoso R, Rothermel BA, et al. Cardiovascular autophagy: concepts, controversies, and perspectives. Autophagy 2013;9:1455-66.

4. Zimetbaum P, Goldman AJC. Ambulatory Arrhythmia Monitoring: Choosing the Right Device. Circulation 2010;122:1629-36.

5. Kokubo Y, Matsumoto CJCJ. Traditional Cardiovascular Risk Factors for Incident Atrial Fibrillation. Circ J 2016;80:2415-22.

6. Mazaris S, Siasos G, Oikonomou E, et al. Atrial Fibrillation: Biomarkers determining prognosis. Curr Med Chem 2019;26:909-15.

7. Thomas G, Moffatt P, Salois P, et al. Osteocrin, a novel bone-specific secreted protein that modulates the osteoblast phenotype. J Biol Chem 2003;278:50563-71.

8. Nishizawa H, Matsuda M, Yamada Y, et al. Musclin, a novel skeletal muscle-derived secretory factor. J Biol Chem 2004;279:19391-5.

9. Asanin M, Stankovic S, Mrdovic I, et al. B-type natriuretic peptide predicts new-onset atrial fibrillation in patients with ST-segment elevation myocardial infarction treated by primary percutaneous coronary intervention. Peptides 2012;35:74-7.

10. Miyazaki T, Otani K, Chiba A, et al. A New Secretory Peptide of Natriuretic Peptide Family, Osteocrin, Suppresses the Progression of Congestive Heart Failure After Myocardial Infarction. Circ Res 2018;122:742-51.

11. Kita S, Nishizawa H, Okuno Y, et al. Competitive binding of musclin to natriuretic peptide receptor 3 with atrial natriuretic peptide. J Endocrinol 2009;201:287-295.

12. Mandalenakis Z, Eriksson H, Welin L, et al. Atrial natriuretic peptide as a predictor of atrial fibrillation in a male population study. The Study of Men Born in 1913 and 1923. Int J Cardiol 2014;171:44-8.

13. Yoshida N, Okamoto M, Hirao H, et al. High plasma human atrial natriuretic peptide and reduced transthoracic left atrial appendage wall-motion velocity are noninvasive surrogate markers for assessing thrombogenesis in patients with paroxysmal atrial fibrillation. Echocardiography 2014;31:965-71.

14. Hodgson-Zingman DM, Karst ML, Zingman LV, et al. Atrial natriuretic peptide frameshift mutation in familial atrial fibrillation. N Engl J Med 2008;359:158-65.

15. Dickey DM, Yoder AR, Potter LR. A familial mutation renders atrial natriuretic Peptide resistant to proteolytic degradation. J Biol Chem 2009;284:19196-202.

16. Hijazi $Z$, Wallentin L. Natriuretic peptide should be used as a routine tool for evaluation of patients with atrial fibrillation. Heart 2019;105:353-4.

17. Subbotina E, Sierra A, Zhu Z, et al. Musclin is an activitystimulated myokine that enhances physical endurance. Proc Natl Acad Sci U S A 2015;112:16042-7.

18. Elliott AD, Linz D, Mishima R, et al. Association between physical activity and risk of incident arrhythmias in 402 406 individuals: evidence from the UK Biobank cohort. Eur Heart J 2020;41:1479-86.

19. Chen WJ, Liu Y, Sui YB, et al. Positive association between musclin and insulin resistance in obesity: evidence of a human study and an animal experiment. Nutr Metab (Lond) 2017;14:46.

20. Li YX, Kai-Chun C, Akihiro A, et al. Role of Musclin in the Pathogenesis of Hypertension in Rat. PLoS One 2013;8:e72004.

21. Calkins H, Hindricks G, Cappato R, et al. WITHDRAWN: 2017 HRS/EHRA/ECAS/APHRS/ SOLAECE Expert Consensus Statement on Catheter and Surgical Ablation of Atrial Fibrillation. Heart Rhythm 2017;14:e275-444.

22. Montalescot G, Sechtem U, Achenbach S, et al. 2013 ESC guidelines on the management of stable coronary artery disease: the Task Force on the management of stable coronary artery disease of the European Society of Cardiology. Eur Heart J 2013;34:2949-3003.

23. Ogawa S, Shinohara Y, Kanmuri K. Safety and efficacy of the oral direct factor xa inhibitor apixaban in Japanese patients with non-valvular atrial fibrillation. -The ARISTOTLE-J study. Circ J 2011;75:1852-9.

24. Finlay MC, Hunter RJ, Baker V, et al. A randomised comparison of Cartomerge vs. NavX fusion in the catheter ablation of atrial fibrillation: the CAVERN Trial. Journal of interventional cardiac electrophysiology : an international journal of arrhythmias and pacing. J Interv Card Electrophysiol 2012;33:161-9.

25. Lip GY, Nieuwlaat R, Pisters R, et al. Refining clinical risk stratification for predicting stroke and thromboembolism 
in atrial fibrillation using a novel risk factor-based approach: the euro heart survey on atrial fibrillation. Chest 2010;137:263-72.

26. Chierchia GB, Di Giovanni G, Ciconte G, et al. Secondgeneration cryoballoon ablation for paroxysmal atrial fibrillation: 1-year follow-up. Europace 2014;16:639-44.

27. Yasui A, Nishizawa H, Okuno Y, et al. Foxo1 represses expression of musclin, a skeletal muscle-derived secretory factor. Biochemical and biophysical research communications. Biochem Biophys Res Commun 2007;364:358-365.

28. Guo Q, Hu H, Liu X, et al. C/EBP $\beta$ mediates palmitateinduced musclin expression via the regulation of PERK/ ATF4 pathways in myotubes. Am J Physiol Endocrinol Metab 2019;316:E1081-92.

29. Gu N, Guo Q, Mao K, et al. Palmitate increases musclin gene expression through activation of PERK signaling pathway in C2C12 myotubes. Biochem Biophys Res Commun 2015;467:521-6.

30. Potter LR, Abbey-Hosch S, Dickey DM. Natriuretic peptides, their receptors, and cyclic guanosine monophosphate-dependent signaling functions. Endocr Rev 2006;27:47-72.

31. Pinnick KE, Nicholson G, Manolopoulos KN, et al. Distinct developmental profile of lower-body adipose tissue defines resistance against obesity-associated metabolic complications. Diabetes 2014;63:3785-97.

32. Patton KK, Ellinor PT, Heckbert SR, et al. N-terminal pro-B-type natriuretic peptide is a major predictor of the development of atrial fibrillation: the Cardiovascular Health Study. Circulation 2009;120:1768-74.

33. Hussein AA, Saliba WI, Martin DO, et al. Plasma B-type natriuretic peptide levels and recurrent arrhythmia after successful ablation of lone atrial fibrillation. Circulation 2011;123:2077-82.

34. Miyazaki T, Otani K, Chiba A, et al. A New Secretory Peptide of Natriuretic Peptide Family, Osteocrin, Suppresses the Progression of Congestive Heart Failure After Myocardial Infarction. Circ Res 2018;122:742-51. 35. Jeremic N, Chaturvedi P, Tyagi SC. Browning of White

Cite this article as: Zhong Y, Zhang J, Tang K, Kou W, Xu S, Yang H, Liu L, Luan P, Mohammed AQ, Abdu FA, Zhao D, Li H, Peng W, Xu Y. Decreased plasma musclin levels are associated with potential atrial fibrillation in non-diabetic patients. Ann Transl Med 2021;9(3):203. doi: 10.21037/atm-203259
Fat: Novel Insight Into Factors, Mechanisms, and Therapeutics. J Cell Physiol 2017;232:61-8.

36. Schafer C, Moore V, Dasgupta N, et al. The Effects of PPAR Stimulation on Cardiac Metabolic Pathways in Barth Syndrome Mice. Front Pharmacol 2018;9:318.

37. Majumder A, Singh M, George AK, et al. Remote ischemic conditioning as a cytoprotective strategy in vasculopathies during hyperhomocysteinemia: An emerging research perspective. J Cell Biochem 2019;120:77-92.

38. Garnvik LE, Malmo V, Janszky I, et al. Physical activity, cardiorespiratory fitness, and cardiovascular outcomes in individuals with atrial fibrillation: the HUNT study. Eur Heart J 2020;41:1467-75.

39. Chen WJ, Liu Y, Sui YB, et al. Increased circulating levels of musclin in newly diagnosed type 2 diabetic patients. Diab Vasc Dis Res 2017;14:116-21.

40. Martins RP, Hamon D, Cesari O, et al. Safety and efficacy of a second-generation cryoballoon in the ablation of paroxysmal atrial fibrillation. Heart Rhythm 2014;11:386-93.

41. Zhuang J, Yi W, Kai T, et al. Association between left atrial size and atrial fibrillation recurrence after single circumferential pulmonary vein isolation: a systematic review and meta-analysis of observational studies. Europace 2012;14:638-45.

42. Arora S, Lahewala S, Tripathi B, et al. Causes and Predictors of Readmission in Patients With Atrial Fibrillation Undergoing Catheter Ablation: A National Population-Based Cohort Study. J Am Heart Assoc 2018;7:e009294.

43. Latchamsetty R, Morady F. Atrial Fibrillation Ablation. Annu Rev Med 2018;69:53-63.

44. Latini R, Masson S, Pirelli S, et al. Circulating cardiovascular biomarkers in recurrent atrial fibrillation: data from the GISSI-atrial fibrillation trial. J Intern Med 2011;269:160-71.

45. Zhang Y, Chen A, Song L, et al. Association Between Baseline Natriuretic Peptides and Atrial Fibrillation Recurrence After Catheter Ablation. Int Heart J 2016;57:183-9. 